\title{
DALLA SEROTONINA E DOPAMINA ALLA TERAPIA DELL'OBESITÀ
}

\author{
MICHELE O. CARRUBA (*)
}

\begin{abstract}
SunTO. - Negli ultimi tre decenni abbiamo seguito un percorso di ricerca che ha tenuto conto, volta per volta, dei risultati che si stavano ottenendo con la continua evoluzione della farmacologia. Si è iniziato con gli studi a livello cerebrale sul ruolo dei sistemi serotoninergici e dopaminergici implicati nel controllo del comportamento alimentare, utilizzando gli approcci della farmacologia classica (somministrazione di farmaci stimolatori o inibitori e osservazione delle modificazioni comportamentali a questi conseguenti). Il passo successivo è stato quello di tentare un approccio più fisiologico per l'approfondimento degli studi sulla serotonina, mediante la modulazione dei circuiti nervosi con specifiche diete. Questo approccio ha dato il via a una nuova concezione della farmacologia nutrizionale, soprattutto nel campo dei disturbi dell'alimentazione. Ci siamo poi concentrati sullo studio dell'obesità come malattia e su un nuovo, e inizialmente molto discusso, approccio multifattoriale che, oltre all'utilizzo di farmaci, prevede l'integrazione di diverse dimensioni biologiche e psicologiche, come la modificazione degli stili di vita e l'educazione culturale, nella terapia dell'obesità stessa e delle malattie correlate.
\end{abstract}

$$
* * *
$$

ABSTRACT. - Following development of pharmacological science in the last three decades, we started with brain studies aimed to characterize the role of serotonin and dopamine systems in feeding behaviour, by using traditional pharmacological approaches, such as injection of stimulatory and/or inhibitory drugs and monitoring the consequent behavioral changes. Then, we tried more physiological approaches to study serotonin system, by modulating its effects through special diets, thus proposing a new concept in nutritional pharmacology, in particular in the treatment of feeding disorders. Then, we focused to the study of obesity, by highlighting the multi-factorial approaches that, in addition to drugs, require an integration of other biological and psychological dimension, such as life style changes and cultural education, in treatment of this and related disorders.

(*) Dipartimento di Biotecnologie Mediche e Medicina Traslazionale, Università degli Studi di Milano, Italia. E-mail: michele.carruba@unimi.it 


\section{INTRODUZIONE}

Prima di iniziare a descrivere alcune attività di ricerca svolte durante la mia carriera, ci tengo a spendere qualche parola riguardo al rapporto che si è instaurato con il Professor Mantegazza. Arrivato da Roma nel lontano 1968, mi stabilii a Milano su invito del Professor Trabucchi, dedicandomi allo studio della placca neuromuscolare a livello del diaframma (campo in cui Mantegazza era considerato un esperto). Iniziarono i primi contatti con Mantegazza che all'epoca insegnava a Pavia, il quale, quasi come un padre, mi dispensava ottimi consigli per condurre al meglio le mie ricerche. Nel 1970 fu trasferito a Milano e da lì iniziò una vera e propria collaborazione diventando l'assistente del professor Mantegazza. Erano gli anni della scoperta della dopamina, del premio nobel per gli studi sulle catecolamine. Ebbi la possibilità di passare 2 anni a Basilea nei laboratori della Hoffmann-La Roche dove si stavano studiando la 6-idrossi Dopamina, una molecola capace di distruggere i neuroni catecolaminergici, e il neurotrasmettitore Serotonina scoperta da Vittorio Espamer nel 1935 a Roma.

\section{BILANCIAMENTO DOPAMINA-SEROTONINA E SQUILIBRI COMPORTAMENTALI}

Primi studi pubblicati nel 1969 avevano messo in luce una correlazione tra la serotonina e il comportamento. Somministrazioni sistematiche della molecola p-clorofenilalanina modificavano il comportamento sessuale dei ratti aumentandone l'attività [1]. Questi aspetti risultavano molto rilevanti per la comprensione del ruolo della serotonina nel cervello e il ruolo delle catecolamine come la dopamina. A tal proposito era utilizzata la molecola 6-idrossi dopamina, che è una molecola capace di distruggere tutti i neuroni catecolaminergici. Il vantaggio del lavoro all'interno di un'azienda farmaceutica e gli insegnamenti di Mantegazza sul rapporto tra struttura chimica di una molecola e la sua attività biologica, hanno permesso di sintetizzare una nuova molecola, la 5,6-diidrossitriptamina (5,6-DHT) e di studiarne gli effetti. Questa molecola che, come la 6-idrossi dopamina aveva un ossidrile in più rispetto al neurotrasmettitore, era in grado di distruggere tutti i neuroni serotoninergici permettendo di studiare la funzione della serotonina nel cervello. Somministrazioni di 5,6-DHT nei ratti provocava disturbi 
comportamentali a livello sessuale, permettendo di identificare il ruolo della serotonina come tono inibitorio sul comportamento sessuale. L'eliminazione quindi di questo tono inibitorio, distruggendo il sistema serotoninergico, disinibiva l'animale causando un comportamento iper sessuale. Nello stesso periodo si cercava di capire anche il ruolo degli altri neurotrasmettitori come per esempio la dopamina. Somministrazioni di L-dopa, che è un precursore della dopamina, davano luogo a effetti simili alla mancanza di serotonina[2]. Questo ha dimostrato l'importanza del giusto equilibrio tra dopamina e serotonina sull'effetto comportamentale.

\section{APPROCCI FISIOLOGICI NEL CONTROLLO DEL COMPORTAMENTO}

Rientrato dall'esperienza di Basilea, con il Professor Mantegazza abbiamo pensato all'utilizzo di un approccio più fisiologico rispetto alla somministrazione di un farmaco. Si è approfondito il caso della serotonina, la quale è sintetizzata a partire da un aminoacido precursore che è il triptofano. In natura esistono alimenti privi di questo aminoacido e quindi si è pensato di effettuare un test in cui i ratti venivano alimentati utilizzando farina di mais, alimento noto per l'assenza di triptofano, per vedere se si aveva una riduzione dell'attività del sistema serotoninergico. I primi test utilizzando questa dieta non mostravano alcun tipo di cambiamento nel comportamento degli animali. Ancora una volta si è dimostrata fondamentale l'esperienza e i consigli del professor Mantegazza, il quale mi fece notare come la farina di mais fosse scarsamente digeribile e quindi la mancanza di triptofano non mostrava alcun effetto in quanto anche il resto degli aminoacidi risultava assente, generando un deficit generalizzato e non uno sbilanciamento rispetto al triptofano. Allora si è pensato di cuocere la farina di mais, processo che permette la liberazione degli aminoacidi rendendoli disponibili per l'assorbimento. In questo modo gli aminoacidi assorbiti erano in grado di raggiungere il cervello e generare un forte sbilanciamento rispetto al triptofano provocando un deficit di serotonina[3]. Anche in questo caso si sono riscontrati gli stessi effetti visti utilizzando il farmaco 5,6diidrossitriptamina, con una riduzione della serotonina e un comportamento iper sessuale dei ratti[4]. L'utilizzo di questo approccio fisiologico ha dimostrato per la prima volta che attraverso l'alimentazione è possibile modificare il biochimismo cerebrale e quindi anche il compor- 
tamento animale. Questo lavoro è stato molto citato in quanto ha segnato una svolta nello studio dei comportamenti e dei disturbi alimentari avendo un grande riscontro nel trattamento di disturbi alimentari come l'anoressia che inizialmente si cercava di curare utilizzando esclusivamente la psicoterapia. L'approccio psicoterapico spesso non aveva alcuna efficacia, in quanto i pazienti affetti da questo disturbo si trovavano in deficit alimentare che ne condizionava il comportamento. Iniziando un trattamento di rialimentazione, si osservava un effetto positivo sulla risposta alla psicoterapia in quanto, soprattutto a livello celebrale, venivano ripristinati i corretti equilibri dei vari aminoacidi precursori dei neurotrasmettitori.

\section{TERAPIA DELL'OBESITÀ E NUOVI APPROCCI}

Nel proseguimento degli studi sui comportamenti, si iniziò con lo studio di alcune molecole che avevano proprietà anoressizzanti come ad esempio la molecola chiamata Mazindol[5][6]. Questa nuova molecola doveva essere studiata per poterla poi utilizzare nella terapia dell'obesità, e come ogni nuova molecola doveva essere comparata ai vecchi farmaci già in uso. Stiamo parlando di amfetamina e fenfluramina che erano i principali farmaci utilizzati per il trattamento dell'obesità, ma di cui non si conoscevano ancora i meccanismi d'azione. Approfondendo lo studio del Mazindol, che presentava gli stessi effetti dell'anfetamina, si sono scoperti anche i meccanismi d'azione di anfetamina e fenfluramina. L'anfetamina ha un'azione sull'attività del sistema dopaminergico inibendo la fame, mentre la fenfluramina è un agonista serotoninergico e favorisce l'insorgere dei segnali di sazietà. Grazie a un semplice esperimento è stato quindi possibile identificare l'azione dei due principali farmaci anoressizzanti allora utilizzati. Ad animali tenuti a digiuno per 24 ore veniva risomministrato il cibo osservando il loro comportamento a seconda del tipo di trattamento a cui erano stati sottoposti; rispetto ai controlli che iniziavano immediatamente ad alimentarsi, gli animali trattati con anfetamina non si alimentavano o lo facevano con notevole ritardo mentre quelli trattati con fenfluramina iniziavano immediatamente a mangiare ma terminavano prima dei controlli per l'attivazione dei segnali di sazietà[7].

Continuarono gli studi sul tema dell'obesità con la scoperta di nuovi farmaci e di nuovi approcci. L'esperienza maturata ci rese cultori 
in materia di farmaci anoressizzanti e terapia dell'obesità, spingendoci nel 1981 a scrivere un libro: "Obesità. Analisi e terapia" [8]. In questo libro è messo in risalto come l'approccio al problema dell'obesità debba essere multifattoriale coinvolgendo la dieta, l'esercizio fisico e la psicologia. Quest'ultimo aspetto ha reso il libro molto discusso soprattutto da parte dei clinici medici che riconoscevano l'obesità unicamente come un problema fisico e non legato alla psicologia, cosa che invece oggi si prende molto in considerazione in quanto il paziente oltre a essere curato va anche rieducato a seguire uno stile alimentare corretto.

Nel 1987 vinsi la cattedra a Brescia e misi in piedi un nuovo laboratorio dove si cominciò ad analizzare il problema dell'obesità da un altro punto di vista. Era stato ormai studiato tutto quello che si poteva studiare sul cervello, e si è iniziato a pensare che il cervello dirige il comportamento alimentare e il dispendio energetico sulla base di una serie di informazioni che gli arrivano dalla periferia.

\section{TESSUTO ADIPOSO E ATTIVITÀ MITOCONDRIALE}

Sebbene anche stomaco e intestino siano in grado di produrre segnali che coinvolgono serotonina e dopamina, concentrammo le nostre attenzioni sul tessuto adiposo, un insieme di cellule adibite all'immagazzinamento di grassi. In particolare fu preso in esame il tessuto adiposo bianco e bruno poi considerato come un organo endocrino in quanto è in grado di produrre alcune sostanze come ad esempio insulina, PAI-1, adispsina, angiotensina, leptina e resistina con capacità di interferire con la funzionalità di altri organi e apparati. Queste interferenze possono dar luogo a patologie come ipertensione, aterosclerosi, diabete e infiammazione cronicha[9][10], tutte patologie tipiche del paziente obeso. Nello studio del tessuto adiposo fu inoltre scoperto un fattore chiamato TNF- $\alpha$ prodotto soprattutto dal tessuto adiposo viscerale e capace di inibire l'espressione dell'enzima eNOS (ossido nitrico sintasi) fondamentale nella mitocondriogenesi[11][12]. Il mitocondrio è quell'organello cellulare adibito alla produzione di energia indispensabile alla cellula per il suo mantenimento e per lo svolgimento delle sue attività. Questa energia è prodotto sotto forma di ATP partendo da glucosio e da acidi grassi. Nei pazienti obesi si riscontra un alto livello di TNF- con conseguente riduzione nel numero di mitocondri che non sono più in grado di bruciare tutti gli acidi grassi circolanti e determi- 
nandone un ulteriore accumulo. Inoltre la scarsa efficienza dei mitocondri nel produrre ATP innesca una serie di segnali al cervello che stimola l'organismo a mangiare ancora di più per sopperire alla mancanza di energia, dando inizio ad un circolo vizioso da cui è molto difficile uscire se non integrando diversi approcci medici e psicologici.

\section{RESTRIZIONE CALORICA, ESERCIZIO FISICO E DIETA SUPPLEMENTATA}

Uno strumento che era utilizzato per la cura dell'obesità era la restrizione calorica nota anche per avere effetti sull'allungamento dell'aspettativa di vita in tutte le specie animali. Iniziammo allora a studiare la dieta per capire se la restrizione calorica avesse effetti sulla mitocondriogenesi scoprendo che essa aumentava l'attività della eNOS e quindi la formazione di NO che riattivava la mitocondriogenesi. Il meccanismo per cui la dieta è in grado di allungare la vita prevenendo tutte quelle patologie tipiche dell'obesità, è anch'esso legato all'espressione di eNOS e alla mitocondriogenesi, permettendoci anche di effettuare dei parallelismi tra obesità e invecchiamento in quanto entrambe coinvolgono gli stessi meccanismi[13]. Un altro meccanismo in grado di attivare l'ossido nitrico, e quindi la mitocondriogenesi, risulta essere l'esercizio fisico il quale insieme alla dieta costituisce uno degli approcci fondamentali per la cura e la prevenzione dell'obesità[14].

Mentre un tempo si cercava di comprendere i meccanismi molecolari attraverso l'utilizzo di farmaci, nel nostro caso il meccanismo eziopatogenetico che porta all'obesità era ben chiaro. È stato trovato che una supplementazione di aminoacidi a catena ramificata somministrata a topi già anziani, è in grado di ridurre l'aumento di peso e di aumentare l'aspettativa di vita del $15 \%$ andando ad agire sui meccanismi di mitocondriogenesi[15]. Inoltre abbiamo dimostrato come l'effetto di questi aminoacidi ramificati sulla mitocondriogenesi sia mediato da eNOS in quanto topi knock out per eNOS non rispondono agli effetti della supplementazione aminoacidica.

\section{CONCLUSIONI}

Ho trascorso molti anni nel campo della ricerca, e ho avuto la possibilità ed il piacere di conoscere moltissime persone brillanti con cui 
poter lavorare e confrontarmi. Più che una conclusione sui lavori che ho descritto in questo capitolo, mi permetto di fare una riflessione. La farmacologia così come la medicina e tutte le altre discipline devono svilupparsi e avanzare con una mentalità sempre aperta a nuove teorie e sempre seguendo le esigenze di una società in continuo cambiamento. Faccio ovviamente riferimento all'esempio del mio libro sull'approccio multifattoriale nella terapia dell'obesità, inizialmente discusso riguardo all'utilizzo della psicoterapia associata alla terapia clinica, ma oggi invece di fondamentale importanza per un percorso efficace e duraturo nel tempo. L'obesità è un problema che sta diventando sempre più rilevante anche in Italia, il paese famoso per la dieta mediterranea; la dieta salutistica per eccellenza, paese che nonostante questo sta arrivando a toccare il 50\% della popolazione obesa o in sovrappeso. Il cambiamento della società, degli stili di vita, il lusso di disporre di cibo in abbondanza e l'abuso che spesso ormai si fa con esso hanno portato all'insorgere di problematiche di impatto rilevante sulla salute della popolazione. Nonostante questo sia un dato di fatto, nonostante l'obesità sia uno stato di palese identificazione visiva oltre alla sua associazione con numerose patologie che indubbiamente richiedono un notevole carico sul sistema sanitario, tutt'ora l'obesità non è riconosciuta come una vera e propria patologia ma solo come un problema estetico. Il mio auspicio è quello che la società si renda conto al più presto della gravità della situazione a cui stiamo andando incontro. E' necessario razionalizzare il proprio stile di vita e la propria alimentazione per poter vivere più a lungo e soprattutto in salute.

\section{BIBLIOGRAFIA}

[1] M.H. Sheard, "The effect of p-chlorophenylalanine on behavior in rats: relation to brain serotonin and 5-hydroxyindoleacetic acid.," Brain Res., vol. 15, no. 2, pp. 524-8, Oct. 1969.

[2] M. Da Prada, M. Carruba, A. Saner, A. O'Brien, and A. Pletscher, "The action L-dopa on sexual behaviour of male rats.," Brain Res., vol. 55, no. 2, pp. 383-9, Jun. 1973.

[3] F. Zambotti, M. Carruba, L. Vicentini, and P. Mantegazza, "Selective effect of a maize diet in reducing serum and brain tryptophan contents and blood and brain serotonin levels.," Life Sci., vol. 17, no. 11, pp. 1663-9, Dec. 1975.

[4] M.O. Carruba, G.B. Picotti, E. Genovese, and P. Mantegazza, "Stimulatory effect of a maize diet on sexual behaviour of male rats.," Life Sci., vol. 20, no. 1, pp. 159-64, Jan. 1977. 
[5] F. Zambotti, M.O. Carruba, F. Barzaghi, L. Vicentini, A. Groppetti, and P. Mantegazza, "Behavioural effects of a new non-phenylethylamine anorexigenic agent: mazindol.,” Eur. J. Pharmacol., vol. 36, no. 2, pp. 405-12, Apr. 1976.

[6] M.O. Carruba, A. Groppetti, P. Mantegazza, L. Vicentini, and F. Zambotti, "Effects of mazindol, a non-phenylethylamine anorexigenic agent, on biogenic amine levels and turnover rate.," Br. J. Pharmacol., vol. 56, no. 4, pp. 431-6, Apr. 1976.

[7] M.O. Carruba, G.B. Picotti, F. Zambotti, and P. Mantegazza, "Mazindol and amphetamine as inhibitors of the uptake and releasers of $3 \mathrm{H}$-dopamine by rat striatal synaptosomes.," Naunyn. Schmiedebergs. Arch. Pharmacol., vol. 298, no. 1, pp. 1-5, May 1977.

[8] Michele Carruba and Paolo Mantegazza, Obesità. Analisi e terapia. Edizioni Minerva Medica 1984.

[9] C.J. Lyon, R.E. Law, and W.A. Hsueh, "Minireview: adiposity, inflammation, and atherogenesis.," Endocrinology, vol. 144, no. 6, pp. 2195-200, Jun. 2003.

[10] P. Trayhurn and I.S. Wood, "Adipokines: inflammation and the pleiotropic role of white adipose tissue.," Br. J. Nutr., vol. 92, no. 3, pp. 347-55, Sep. 2004.

[11] A. Valerio et al., "TNF-alpha downregulates eNOS expression and mitochondrial biogenesis in fat and muscle of obese rodents.," J. Clin. Invest., vol. 116, no. 10, pp. 2791-8, Oct. 2006.

[12] E. Nisoli et al., "Mitochondrial biogenesis in mammals: the role of endogenous nitric oxide.," Science, vol. 299, no. 5608, pp. 896-9, Feb. 2003.

[13] E. Nisoli et al., "Calorie restriction promotes mitochondrial biogenesis by inducing the expression of eNOS.," Science, vol. 310, no. 5746, pp. 314-7, Oct. 2005.

[14] R. Vettor et al., "Exercise training boosts eNOS-dependent mitochondrial biogenesis in mouse heart: role in adaptation of glucose metabolism.," Am. J. Physiol. Endocrinol. Metab., vol. 306, no. 5, pp. E519-28, Mar. 2014.

[15] G. D'Antona et al., "Branched-chain amino acid supplementation promotes survival and supports cardiac and skeletal muscle mitochondrial biogenesis in middle-aged mice.," Cell Metab., vol. 12, no. 4, pp. 362-72, Oct. 2010. 